\title{
Le débat entre naturalisme et nominalisme dans quelques pièces de Shakespeare
}

Jean-Jacques Chardin

\section{(2) OpenEdition}

\section{Journals}

Édition électronique

URL : http://journals.openedition.org/shakespeare/897

DOI : 10.4000/shakespeare.897

ISSN : 2271-6424

Éditeur

Société Française Shakespeare

Édition imprimée

Date de publication : 1 novembre 2002

Pagination : 19-30

Référence électronique

Jean-Jacques Chardin, « Le débat entre naturalisme et nominalisme dans quelques pièces de

Shakespeare ", Actes des congrès de la Société française Shakespeare [En ligne], 19 | 2002, mis en ligne le 01 novembre 2007, consulté le 04 mai 2019. URL : http://journals.openedition.org/shakespeare/897 ; DOI : 10.4000/shakespeare.897 


\section{S H A K E S P E A R E \\ \& L E M O Y E N - Â G E}

Société Française Shakespeare

Actes du Congrès de 2001

* * *

Textes réunis et présentés par

Patricia DORVAL

publiés sous la direction de Jean-Marie MAGUIN 


\section{LE DÉ B A T EN TRE NA T URALIS M E E T NOM INALIS ME DANS QUELQUES P I È C S D E S H A K E S P A R E}

La réflexion sur la langue fait partie de l'horizon culturel de la Renaissance. Que l'on songe au rôle de la rhétorique dans l'éducation ou à l'interprétation nouvelle et souvent ambiguë des textes sacrés par. les penseurs de la Réforme, l'origine, la nature, le fonctionnement du langage sont des enjeux épistémologiques majeurs de la pensée du XVIe siècle. Il est communément admis que la théorie linguistique dominante à l'époque est celle qui consiste à considérer le signe linguistique comme icône ou représentation fidèle de la chose signifiée. Mahood résume cette idée dans le dernier chapitre de son Shakespeare's Wordplay (1957):

Like Plato, the Elizabethans believed in the truth of names, but whereas, according to Socrates in the Cratylus, these right names had been given by «the legislators», to sixteenthcentury ways of thinking the right names of things had been given by God and found out by Adam. ${ }^{1}$

Cette conception dite «naturaliste», ou «réaliste» ou encore maladroitement «cratylienne» de la langue considérait les mots comme motivés de façon naturelle et porteurs de significations univoques. Keir Elam ajoute pour sa part dans Shakespeare's Universe of Discourse que la spéculation 
néoplatonicienne de la Renaissance, avec l'Argumentum in Cratylum de Ficin et les Thèses de Pic de la Mirandole, appuyée sur une lecture hâtive et fautive du Cratyle, contribua à formaliser l'idée que la langue était fondée sur le principe de la motivation naturelle des signes :

Semantic naturalism - and the mystical and occult theories of the sign that often went with it - found their most authoritative support in the Renaissance in a mistakenly literal reading of the Cratylus. ${ }^{2}$

Des recherches plus récentes, parmi lesquelles celles de Marie-Luce Demonet $^{3}$, montrent à l'inverse que s'affirme à la Renaissance une théorie linguistique qui tend à penser le signe comme distinct de la chose signifiée, comme item dont le sens est attribué par convention. «Le nom n'est rien d'autre», écrit Rodolphe Agricola dans le De Inventione Dialectica (1515), «qu'un mot prononcé, institué par convention entre les hommes pour signifier une chose» ${ }^{4}$. Montaigne fait écho à Agricola dans les premières lignes de son chapitre sur la gloire : «Il y a le nom et la chose ; le nom, c'est une voix qui remerque et signifie la chose ; le nom, ce n'est pas une partie de la chose, ny de sa substance, c'est une pièce étrangère joincte à la chose et hors d'elle» ${ }^{5}$.

Imprégné encore de dialectique médiévale, Montaigne pense la langue surtout comme discours oral, ce qui lui fait écrire «voix» pour «mot». L'on pourrait citer également d'autres philosophes du langage, parmi lesquels Charles de Bovelles et son Liber de Differentia Vulgarium Linguarum (1533), pour qui la langue est un système de signes arbitrairement liés aux choses signifiées. Cette théorie linguistique conventionnaliste prend appui sur la définition aristotélicienne du signe présentée dans De l'Interprétation :

[...] le nom est un son vocal, possédant une signification conventionnelle, sans référence au temps [...] Signification conventionnelle [...] en ce que rien n'est par nature un nom. ${ }^{6}$

Sans vouloir cautionner une position aussi tranchée que celle de Demonet pour qui «la philosophie du langage à la Renaissance apparaît comme un relais dans une continuité qui va d'Aristote à Saussure» ', il est clair pourtant que le XVIe siècle est le lieu d'un débat, d'une confrontation, 
d'une opposition entre deux conceptions de la langue, opposition qui avait déjà fortement travaillé la pensée médiévale.

L'on sait que les théories naturalistes, pour lesquelles le mot est conçu comme ressemblance de la chose signifiée, avaient déjà été contestées par Abélard et sa réfutation du dogme de la motivation naturelle du signe linguistique (la première page de sa Dialectique reprend la définition aristotélicienne du signe). Et William of Ockham avait pour sa part postulé que le mot est lié à la chose signifiée par pure convention et qu'il n'est en aucun cas l'expression d'une essence supérieure à son existence en tant qu'objet : «In this way, an uttered word, which is really a single quality, is universal ; for it is a conventional sign to signify many things» ${ }^{8}$. L'hypothèse que j'aimerais soutenir est que le théâtre de Shakespeare reprend en le mettant en scène le grand débat médiéval qui opposait les tenants du nominalisme aux partisans d'un naturalisme sémantique sans doute plus canonique.

Un certain nombre de personnages shakespeariens portent des noms qui expriment la quintessence de leur être ou, pour paraphraser et contredire Montaigne, dont le nom est «une partie de la substance». C'est le cas de Thersites, le fou de Troilus and Cressida dont la fonction dans le texte est d'être un observateur critique et distancié des turpitudes du monde. Sa première apparition sur la scène, dans une conversation avec Achilles à propos d'Ajax, est signalée par une allusion directe au voir :

Achill. How now, Thersites, what's the matter man?

Thers. You see him there, do you?

Achill. Ay : what's the matter?

Thers. Nay look upon him.

Achill. So I do: what's the matter?

Thers. Nay but regard him well.

Achill. Well ?-why, so I do.

Thers. But yet you look not well upon him

(Troilus and Cressida, II.1.59-65)

Thersites est bien un oil et l'itération du champ sémantique de la vision souligne l'accord entre le nom et le rôle du voyeur qui lui est dévolu. Thersites est aussi l'esprit de la pièce, le «wit», contre lequel Achilles conseille à Ajax de ne pas se mesurer (Achill. «Will you set your wit to a fool's? - Thers. No I warrant you the fool's will shame it», Troilus and Cressida, II.1.88-9). L'étymologie latine de «wit», de 
«videre», confirme la connexion entre nom, personnage et fonction. Thersites participe à cette scénographie en anamorphose qu'est Troilus and Cressida où les points de vue, les jeux de regard et les perspectives s'enchâssent pour figurer un univers en décentrement.

L'autre fou shakespearien sur lequel je souhaiterais m'attarder un peu, c'est Touchstone dans As You Like It qui lui aussi porte un nom résumant la fonction qu'il assume. Qualifié de «wit» par Celia («How now, wit : whither wander you ?», As You Like It, I.2.53), le nom de Touchstone est en soi un trait d'esprit. Touchstone, c'est la pierre de touche grâce à laquelle, en alchimie, on essaie l'or et l'argent. Et Touchstone est bien le pivot central de la pièce, sa pierre angulaire, contre laquelle s'essaie un moment l'esprit des autres figures du drame, celui du berger Corin par exemple (III.2) qui devra abandonner la partie faute d'un esprit suffisamment aiguisé. Et Celia dit de Touchstone qu'il est «a whetstone» (I.2.52), une pierre à affûter. Marie-Madeleine Martinet rappelle par ailleurs que le mot Touchstone apparaît souvent comme métaphore du miroir dans des titres d'ouvrages comme The Optick Glasse of Humors, or the Touchstone of a Golden Temper de Thomas Walkington (1607) ou encore A Touchstone for the Time, ajout de George Whetstone à A Mirour for Magestrates of Cyties (1584) 9 . Le livre comme miroir, ou pierre de touche, ou encore verre optique, rectifie la vision de celui qui le lit. Par ses jeux sur la langue, Touchstone introduit semblablement dans la pièce des points de vue qui diffractent les données de l'intrigue. Touchstone, comme son nom l'indique, est un instrument d'optique, une perspective par laquelle le regard de biais, de «bon biais» comme l'écrit Montaigne, sert à redresser les formes ou plus exactement les idées.

Recherchant la lisibilité de noms, Shakespeare joue abondamment sur l'onomastique, illustration presque parfaite de ce que l'on pourrait appeler un cratylisme élémentaire. Le nom est juste, il est l'icône de l'objet signifié (ou du personnage désigné) qu'il imite ou révèle au moyen de sons, de lettres et de syllabes. Code signalétique, le nom dessine une géographie des choses et donne à voir ou à entendre une cartographie du moi. Le nom est la signature de l'être et l'on pourrait facilement discerner, à l'arrière-plan de cette adéquation entre la marque linguistique et la chose signifiée, la confirmation de la théorie des correspondances qui fonde encore l'épistémè de la Renaissance et que Michel Foucault a bien décrite dans Les Mots et les choses. Si le langage vaut comme «signe des choses», connaître le mot c'est donc connaître la chose dont il est le miroir, ce qui en filigrane sous-entend bien que le savoir repose sur le principe fondateur de l'analogie. L'onomastique s'inscrit dans la recherche d'une langue 
adamique, originelle et univoque, qui permettrait de déchiffrer le cosmos dans son intégralité.

À cette conception réaliste de la langue se rattache l'idée du pouvoir magique des mots. Si Desdemona refuse avec tant d'obstination de prononcer le mot «whore» et recourt à une périphrase alambiquée (Desd. «Am I that name, Iago ?» Iago. «What name, fair lady ?» Desd. «Such as she says my lord did say I was», Othello, IV.2.119-21), c'est qu'elle confère au nom une force illocutoire magique qui la ferait devenir aux yeux des autres, une putain. Juliet, semblablement, apprenant le départ de Romeo, se dit mise à mort par la puissance du mot «banished»: «To speak that word / Is father, mother, Tybalt, Romeo, Juliet / All slain, all dead» (Romeo and Juliet, III.2.122-4). L'anagramme «words» / «swords», si courante dans le langage de l'époque, atteste bien cette capacité des mots à opérer comme des poignards. Parfois même, une seule syllabe suffit à assassiner: "Say thou but 'Ay' / And that bare vowel ' $I$ ' shall poison more / Than the death-darting eye of cockatrice» (Romeo and Juliet, III.2.45-7) ${ }^{10}$. L'image du poison fonctionne ici comme allusion proleptique à la mort de Romeo et l'ironie dramatique, ressort structurel essentiel de la pièce, surcharge la langue d'une force magique particulière puisque le sens des mots, non directement perçu par le personnage qui les prononce, ici Juliet, est confirmé a posteriori par le cours irrémédiable des événements. L'implacabilité du destin sur lequel repose la tragédie s'accommode de l'idée d'une motivation puissante et naturelle des mots. Faut-il conclure au cratylisme de Shakespeare ? Sans doute que non. Le cratylisme implique la motivation naturelle des signes, c'est-à-dire le rejet de toute intervention humaine dans la justesse du processus de nomination. Tel le législateur du texte de Platon, Shakespeare recourt à des noms justes pour les besoins de sa fiction. Et si Juliet croit à la magie des sons et à la puissance des mots, elle conteste dans le même temps la capacité naturelle des noms à signifier.

Le nom de Romeo inspire à Juliet une célèbre méditation sur l'arbitraire du signe :

What's in a name? That which we call a rose

By any other word would smell as sweet ;

So Romeo would, were he not Romeo call'd,

Retain that dear perfection which he owes

Without that title.

(Romeo and Juliet, II.2.43-6) 
Le nom n'a pas par nature la capacité de signifier, il est un vide dont le rapport à la chose est strictement conventionnel. Lorsque Falstaff dans 1 Henry IV médite sur l'honneur pour conclure qu'il ne s'agit que d'un mot et que l'essence d'un mot n'est rien d'autre que de l'air ( «What is honour? A word. What is in that word honour? What is that honour? Air», I Henry IV, V.1.134-5), il prolonge l'intuition de Juliet sur l'arbitraire du nom et reprend, sur le mode comique, la théorie du «flatus vocis» de Roscelin de Compiègne, défenseur et propagateur du nominalisme au XIe siècle et pour lequel les noms ne sauraient refléter les propriétés des choses. Romeo and Juliet oscille ainsi entre une spéculation sur le pouvoir des sons et la force des mots héritée en droite ligne de l'argumentation de Cratyle et une réflexion sur la disjonction entre l'item lexical et la réalité qu'il cherche à traduire. Interrogeant les rapports de la langue au monde, Shakespeare reprend sans véritablement le trancher ici le grand débat médiéval entre naturalisme et nominalisme.

Dans Julius Casar, où il est aussi question du pouvoir des mots, Shakespeare met à nouveau en scène les deux conceptions du langage et démontre les dangers d'un système de la langue fondé sur l'analogie. Dans une pièce où le travail sur les signes est mis en avant par les augures, les prodiges et les rêves qui annoncent l'inexorabilité d'un destin d'avance scellé, la récusation d'une motivation naturelle de la langue est opérée sur le mode tragique par la mise à mort du poète Cinna. Cinna, celui dont la fonction est de jouer avec la plasticité de la langue, est assassiné par la foule déchaînée non pas parce qu'il est dangereux ou parce qu'il écrit de mauvais vers, mais parce que son nom est Cinna, et qu'il est l'homonyme du conjuré Lucius Cornellius Cinna. Aux yeux de la foule, porter le nom de Cinna est signe de culpabilité :

3 Plebeian. Your name, sir, truly.

Cinna. Truly, my name is Cinna.

1 Plebeian. Tear him to pieces, he's a conspirator. [...]

Cinna. I am not Cinna the conspirator.

4 Plebeian. It is no matter, his name's Cinna.

Pluck but his name out of his heart and turn

him going.

(Julius Casar, III.3.24-30)

La langue tue, elle est soudainement devenue folle, «Slaying is the word», comme l'affirme Brutus en apprenant la mort de Stratilius à la fin 
de la pièce. L'exigence de vérité que souligne ici la répétition de «Truly», déjà indiquée dès l'ouverture de la pièce dans la discussion entre les deux tribuns, Marullus et Flavius, et les citoyens, est le signe que la cité ne peut supporter l'équivoque. Shakespeare fait ici la démonstration du pouvoir mortifère d'un système de la langue réduit à l'univocité du signe. En fait, si la foule est si prompte à se débarrasser de Cinna, c'est qu'elle a été travaillée à son insu par les discours de Brutus et de Marc Antoine au Forum. Il suffit que les deux orateurs prennent la parole à tour de rôle pour que celle-ci accorde sa créance alternativement à l'un puis à l'autre, sans s'apercevoir qu'elle est l'objet d'une manipulation qui la dépasse. Les réactions instinctives et contradictoires de la foule figurent les dangers d'une adhésion sans faille à un langage perçu instinctivement comme univoque et porteur de vérité. Ce que la pièce nous enseigne alors c'est que le langage «adamique», fondé de fait sur la réification du signe, n'est que l'instrument de la dictature. C'est bien sûr Marc Antoine qui est l'artisan de cette désincarnation de la langue. Confronté au spectacle du corps étendu de César, Marc Antoine considère le cadavre comme «memento mori», image du néant de la mort, signe nu de la fragilité humaine. Marc Antoine donne au signe visuel, à l'icône qu'est devenu le corps de César, la signification la plus directe qui soit, et l'ostentation du cadavre, topos par excellence de l'esthétique baroque, devient en fait pré-texte à lecture univoque. Et la harangue à la foule est pour Marc Antoine l'occasion de littéraliser le meurtre de César, de le retourner à son sens originel, celui d'un crime affreux contre l'ordre. Désallégorisation et degré premier de sémantisation sont les outils rhétoriques mis en œuvre par Marc Antoine. Ce processus de destruction de la valeur allégorique du cadavre qui s'oppose à la lecture sacrificielle du meurtre que fait Brutus, a été bien analysé par Patricia Dorval dans un article consacré à la rhétorique dans Julius Casar".

La grande scène du Forum est construite comme pièce dans la pièce, comme mimodrame, où les orateurs / acteurs jouent un rôle devant un parterre abusé incapable de distinguer le mot de la réalité à laquelle il renvoie. Ce sont exactement les griefs que Prospero, dans The Tempest, nourrit contre son frère Antonio :

He being thus lorded,

Not only with what my revenue yielded, But what my power might else exact, like one Who, having into truth by telling of it, Made such a sinner of his memory To credit his own lie, he did believe He was indeed the duke, out o'th' substitution 
And executing th'outward face of royalty

With all prerogative. His ambition growing-

[...]

To have no screen between this part he played

And him he played it for, he needs will be

Absolute Milan.

(The Tempest, 1.2.97-107)

Prospero accuse Antonio non seulement d'avoir usurpé son pouvoir mais surtout d'avoir investi les mots d'une réalité qui ne leur appartient pas. Le simple fait de prononcer le mot a fait naître dans l'esprit d'Antonio l'idée qu'il possédait la chose désignée par le mot, en d'autres termes ici la vérité. Antonio est piètre linguiste puisqu'il confond le signe et la chose et Prospero rapproche cette ignorance de l'attitude naïve du spectateur qui confond l'illusion théâtrale et la réalité. La réflexion sur le langage; outre qu'elle mène au rejet d'une vérité donnée comme absolue, se conjugue ici à une spéculation sémiologique plus large qui interroge la nature des signes visuels et leur rapport au réel, sans doute pour indiquer au spectateur que le grand système de signes qu'est le monde ne peut pas se laisser décoder par le principe réducteur de l'analogie.

La transcription des complexités du réel doit s'opérer par le truchement d'une langue irisée, polymorphe et plurivoque comme celle des fous, dont Feste, dans Twelfth Night, est peut-être le meilleur exemple. À la question de Viola concernant sa fonction, Feste répond qu'il est le corrupteur des mots de la comtesse Olivia. Corrompre la langue, c'est sans doute la pervertir en en retournant les sens comme un gant, c'est également rompre le lien supposé entre le signe et la chose pour redonner un ou des sens nouveau $(x)$ «aux mots de la tribu». Feste, par cette pratique linguistique à la fois destructrice et poétique, s'oppose à Andrew Aguecheek que Toby définit pourtant comme un bon linguiste : «He plays o' th' viol-de-gamboys, and speaks three or four languages word for word without book, and hath all the good gifts of nature» (Twelfth Night, I.3.23-5). Empêtré dans la littéralité du signe à laquelle il manifeste une adhésion absolue, jusque dans le passage d'une langue à l'autre, Aguecheek est incapable de décoder le moindre jeu sur les mots. La lecture littérale des signes est aussi ce qui, dans Twelfth Night, caractérise Malvolio qui croit déceler la réalité intrinsèque d'Olivia dans la forme des lettres qu'elle est censée avoir écrites sur la missive : «By my life, this is my lady's hand. These be her very c's, her u's, and her t's, and thus makes she her great P's. It is in contempt of question her hand» (Twelfth Night, II.5.82-5). 
Aveugle aux allusions sexuelles et scatologiques qui ponctuent son discours, Malvolio devient l'objet ridicule d'un attachement fétichiste à la force magique de la langue.

Aguecheek est lui aussi fortement malmené par les sarcasmes de Maria et de Feste qui lui renvoient, dans le miroir des mots, une image de sa stupidité. Il avoue lui-même être privé d'esprit : «Methinks sometimes I have no more wit than a Christian or an ordinary man has ; but I am a great eater of beef, and I believe that does harm to my wit». (Twelfth Night, I.3.79-82). Théorisé par John Locke au XVIIe siècle, l'esprit, «wit», est la mise en relation de pensées ou d'idées disparates par le biais d'un langage imagé dont les tropes assurent la créativité :

Wit lying in the assemblage of ideas, and putting these together with quickness and variety wherein can be found any resemblance or congruity, thereby to make up pleasant pictures and agreeable visions in the fancy. ${ }^{12}$

La définition proposée par Locke rappelle les analyses d'Aristote consacrées à la métaphore, elle aussi chargée de rapprocher syntaxiquement et sémantiquement des objets ou des choses éloignées : «Il faut, comme nous l'avons dit précédemment, tirer ses métaphores de choses appropriées... comme en philosophie, apercevoir des similitudes entre des objets fort distants témoigne d'un esprit sagace» ${ }^{13}$, écrit Aristote dans la Rhétorique. La métaphore, comme l'esprit avec lequel elle partage cette faculté de jouer avec la langue, est un instrument de cognition qui permet d'accéder à une connaissance des dynamismes du réel. Car c'est bien à un réel en mouvement, en «acte», comme le dit Aristote que ressortit cette conception de la métaphore.

Dans Hamlet, pièce écrite presque à la même époque que Twelfth Night, Shakespeare reprend à nouveau la même opposition entre une langue fermée, enroulée sur elle-même et dépositaire d'une vérité qui n'est qu'apparence et un travail plus subversif sur la plurivocité des signes. L'apologiste de la raison qu'est Horatio établit dès l'ouverture de la pièce un rapport d'analogie entre les mots et les choses. Sa rencontre avec le spectre confirme la véracité de la description que Marcellus et Barnardo avaient faite de leur première confrontation avec lui :

This to me

In dreadful secrecy impart they did, And I with them the third night kept the watch, Where, as they had deliver'd, both in time, 
Form of the thing, each word made true and good, The apparition comes.

(Hamlet, I.2.206-11)

Horatio abolit la distance entre le signe linguistique et l'événement, comme si l'apparition n'était que la transcription iconique et muette du discours auparavant prononcé. Raison et vérité s'entrecroisent dans le langage d'Horatio, dont le nom, superposant habilement «ratio» et «oratio», désigne clairement la conception qu'il paraît se faire de la langue. Pourtant dans Hamlet, la vérité est suspecte et la fermeture de la langue imposée par Horatio implose sous l'exercice de sape pratiqué par Hamlet. La paronomase «kin» / «kind» et l'antanaclase «sun» / «son» sur lesquelles joue Hamlet dans la première conversation avec Claudius induisent le fonctionnement oblique d'une langue ouverte à l'ambiguïté. Dans la grande scène avec la mère (III.4), l'image de la dague des mots pénétrant les oreilles de Gertrude ( O speak to me no more. / These words like daggers enter in my ears. / No more, sweet Hamlet», Hamlet, III.4.94-6), souligne sans doute, derrière le rappel du crime contre le vieil Hamlet, le viol métaphorique dont Gertrude est ici la victime et que traduit l'anagramme courante à la Renaissance entre «ears» et «arse». À moins qu'elle ne suggère le processus d'insémination de la langue auquel se livre Hamlet pour découvrir aux yeux de Gertrude des réalités qu'elle ne pouvait ou ne voulait pas entrevoir.

On retrouve une métaphore d'engendrement liée au pouvoir de la langue dans une remarque que Touchstone adresse à la jeune Audrey pour lui reprocher de ne pas savoir décoder ses jeux de mots : «When a man's verses cannot be understood, nor a man's good wit seconded with the forward child, understanding, it strikes a man more dead than a great reckoning in a little room» (As You Like It, III.3.9-12). L'image de l'enfantement, prolongeant le sens sexuel de «wit», souligne l'aspect créateur de l'esprit. «Wit» ensemence la langue qui accouche de sens neufs et se donne comme la traduction langagière d'un réel en perpétuelle évanescence ${ }^{14}$.

Et qu'est-ce que le réel ? Peut-être une accumulation de points de vue, à l'instar de l'île mythique de The Tempest située quelque part entre Tunis et l'Italie et qui n'existe que dans et par la subjectivité des observateurs qui la décrivent. Dans cet univers où tout est jeux de regards et illusions d'optique, le réel n'a finalement pas plus de poids qu'un décor de théâtre. Ce que les pièces de Shakespeare nous disent en dernière analyse, c'est que le pouvoir des mots réside moins dans leur capacité à nommer les choses, 
les mots peuvent même exister sans les choses, qu'à faire rêver, à créer des images mentales et des fictions qui n'entretiennent avec le réel qu'un rapport finalement lointain.

Comme le héros du Songe de Poliphile que Logistique et Thélémie promènent dans le jardin des signes, le spectateur des pièces de Shakespeare est invité à une pérambulation dans l'univers des mots. La spéculation linguistique que déploient les textes, d'un cratylisme d'école à une réflexion sans doute plus angoissée sur l'inanité des noms et la fracture entre le mot et le référent, est indissociable d'une vision du monde et d'une conception particulière du réel. Les apports de la science nouvelle et le développement des instruments d'optique vont progressivement reléguer au rang d'illusions les visions figées et hiérarchisées du cosmos tout en projetant la pensée vers l'effrayante béance d'une possible disjonction entre l'homme et Dieu. En fait, c'est toute une épistémè du même, de la réduplication et de l'unitaire qui est alors mise à distance et que le fonctionnement de la langue, appuyé sur des conceptions divergentes, s'efforce de véhiculer. Mais ce regard neuf est nourri de savoir ancien ; il avait été préparé par le Moyen-Âge, non pas le Moyen-Âge néoplatonicien, mais le Moyen-Âge rátionaliste, celui de Roscelin, d'Abélard ou d'Ockham qui avaient en leur temps contesté la prééminence de la chose en tant que réalité ontologique pour laquelle le nom n'aurait été qu'image ou miroir, et dont la lecture du monde, démarquée déjà du dogme des analogies naturelles, repose sur ce qu'Umberto Eco appelle «un tissu rhizomatique de propriétés [...] culturelles» ${ }^{15}$. La signifiance est de l'ordre de la coutume, de l'habitude, de la convenance et les sources de la modernité de Shakespeare, tant vantée par la critique, sont peut-être à chercher dans la culture des grands ancêtres. 


\section{NOTES} p. 169 .

${ }^{1}$ M.M. Mahood, Shakespeare's Wordplay (London: Methuen, 1957),

${ }^{2}$ Keir Elam, Shakespeare's Universe of Discourse: Language-Games in the Comedies (Cambridge: Cambridge University Press, 1984), p. 122.

${ }^{3}$ Marie-Luce Demonet, Les Voix du signe : nature et origine du langage à la Renaissance (1480-1580). Thèse Paris XII, 1991.

${ }^{4}$ Rodolphe Agricola, De Inventione Dialectica, 1515 , L.I. $\mathrm{I}^{\circ} \mathrm{C} 4 \mathrm{r}^{\circ}$, in Marie-Luce Demonet, Les Voix du signe: nature et origine du langage à la Renaissance (1480-1580), p. 87.

5 Michel de Montaigne, «De la Gloire», Essais, II, 16 (Paris : Flammarion, 1979), p. 282.

${ }^{6}$ Aristote, De L'Interprétation (Paris : Vrin, 1966), p. 79.

${ }^{7}$ Marie-Luce Demonet, Les Voix du signe : nature et origine du langage à la Renaissance (1480-1580), p. 6.

${ }^{8}$ William of Ockham, Philosophical Writings : A Selection, translated by Philoteus Boehner (Indianapolis: The Library of Liberal Arts, 1934), p. 37.

9 Marie-Madeleine Martinet, Le Miroir de l'esprit dans le théâtre élisabéthain (Paris : Didier Érudition, 1981), p. 76 et p. 220.

${ }^{10} \mathrm{~J}$ 'emprunte cette analyse à Pierre Iselin dans «'What shall I swear by ?' Langue et idolâtrie dans Romeo and Juliet», in Jean-Marie Maguin et Charles Whitworth (eds.), Roméo et Juliette: nouvelles perspectives critiques, Collection Astraa, $\mathrm{n}^{\circ} 5$ (Montpellier: Publications de l'Université de Montpellier III, 1993), p. 165-88.

${ }^{11}$ Patricia Dorval, «Mort de l'allégorie dans Jules César: le sens sacrifié», in Michel Bitot (ed.), Shakespeare : Julius Caesar, Actes du Colloque de Tours (Tours : Publications de l'Université François Rabelais, Tours, 1995), p. 189-204.

${ }^{12}$ John Locke, Essay Concerning Human Understanding, Book II, Chapter IX, «Of Discerning and Other Operations of the Mind» (1690) (London : Everyman Dent, 1961), p. 123.

${ }^{13}$ Aristote, Rhétorique, Livre III, 1412a (Paris: Les Belles Lettres, 1989), p. 68.

14 Je reprends ici une analyse que j'ai faite dans As You Like It ou le palimpseste du sens (Paris : Messene, 1997).

${ }^{15}$ Umberto Eco, Sémiotique et Philosophie du langage (Paris : Presses Universitaires de France, 1988), p. 163. 